\title{
NADH-Dependent Flavin Oxidoreductase of Mycoplasma Hyopneumoniae Functions as a Potential Novel Virulence Factor and not Only as a Metabolic Enzyme
}

\section{Xing Xie}

Jiangsu Academy of Agricultural Sciences

Fei Hao

Jiangsu Academy of Agricultural Sciences

Rong Chen

Jiangsu Academy of Agricultural Sciences

Jingjing Wang

Jiangsu Academy of Agricultural Sciences

Yanna Wei

Jiangsu Academy of Agricultural Sciences

Jin Liu

Wuhan Polytechnic University

Haiyan Wang

Jiangsu Academy of Agricultural Sciences

Zhenzhen Zhang

Jiangsu Academy of Agricultural Sciences

\section{Yun Bai}

Jiangsu Academy of Agricultural Sciences

\section{Guoqing Shao}

Jiangsu Academy of Agricultural Sciences

\section{Qiyan Xiong}

Jiangsu Academy of Agricultural Sciences

Zhixin Feng ( $\sim$ fzxjaas@163.com )

Jiangsu Academy of Agricultural Sciences https://orcid.org/0000-0001-9113-4706

\section{Research}

Keywords: Mycoplasma hyopneumoniae (Mhp), NADH-dependent flavin oxidoreductase, adhesion, pathogenic, virulence factor 
Posted Date: July 27th, 2021

DOl: https://doi.org/10.21203/rs.3.rs-723495/v1

License: (c) (i) This work is licensed under a Creative Commons Attribution 4.0 International License. Read Full License 


\section{Abstract}

Background: Mycoplasma hyopneumoniae (Mhp) is the main pathogen of enzootic pneumonia, pigs infected with Mhp demonstrate mainly poor growth and a reduced feed conversion rate, a disease that has a significant impact on the pig industry and pock production worldwide. The pathogenesis, especially possible virulence factors of Mhp, has still not been fully clarified.

Results: The transcriptome and proteomic analysis of Mhp strains differed in virulence based on reported literature, and RNA transcription expression levels between high- and low-virulence strains initially indicated that nicotinamide adenine dinucleotide (NADH)-dependent flavin oxidoreductase (NFOR) was related to Mhp pathogenicity. Prokaryotic expression and purification of the NFOR protein of Mhp were performed, a rabbit-origin polyclonal antibody of NFOR was prepared, and multiple sequence alignment and evolutionary analysis of Mhp NFOR were performed. For the first time, it was found that the NFOR protein was conserved among all Mhp strains, and NFOR was localized on the cell surface and could adhere to immortalized porcine bronchial epithelial cells (hTERT-PBECs). Adhesion to hTERT-PBECs could be specifically inhibited by an NFOR polyclonal antibody, and the adhesion rates of both high- and lowvirulence strains, 168 and 168L, significantly decreased by more than 40\%. Moreover, Mhp NFOR could not only recognize and interact with host fibronectin and plasminogen but could also induce cellular oxidative stress and apoptosis in hTERT-PBECs. Lactate dehydrogenase release of Mhp NFOR in hTERTPBECs was significantly positively correlated with the virulence of Mhp.

Conclusions: Overall, in addition to being a metabolic enzyme related to oxidative stress, NFOR may also function as a potential novel virulence factor of Mhp, thus contributing to the pathogenesis process of Mhp, providing new ideas and theoretical support for studying the pathogenic mechanisms of other mycoplasmas.

\section{Background}

As the simplest self-replicating organism, Mycoplasma has the smallest genome. It is speculated that they evolved from the degeneration of gram-positive bacteria and are most closely related to certain clostridia in phylogeny [1, 2]. Due to its limited biosynthesis and metabolism capabilities, Mycoplasma relies on infected host cells to provide nutrients to meet the necessary needs of its parasitic life [1], and they have also developed mechanisms to invade and further survive in host cells. The highly plastic variable surface protein group is responsible for the rapid changes of the main surface protein antigens and the invasion of nonphagocytic host cells and the subsequent regulation of the host immune system [3-7]. These mechanisms contribute to the establishment of chronic infection and further to the persistence of Mycoplasma in the host.

Many species of mycoplasmas are pathogens that cause various diseases in livestock production and eventually lead to heavy economic losses [8, 9]. Among them, Mycoplasma hyopneumoniae (Mhp), the pathogen of porcine endemic pneumonia, is a highly contagious and globally distributed porcine 
respiratory disease. It usually destroys the epithelium along with the respiratory tract, making pigs vulnerable to secondary infections by other bacteria and viruses and ultimately causing significant economic losses to the global pig industry [9]. However, a basis for the immune pathogenesis of Mhp has not yet been fully clarified. Some scholars have considered that the onset of porcine enzootic pneumonia depends on Mhp virulence factors, which enable the pathogen to evade the host defense mechanism, and the production of molecules that participate in processes including cell-host adhesion, response to environmental stress of the host, and immune regulation [10]. Therefore, uncovering novel virulence factors would be of great importance in illuminating the mechanism of Mhp pathogenesis.

Despite the presence of surface proteins, a variety of metabolic enzymes have been identified and described as critical pathogenicity determinants not limited to their roles in basic metabolism $[11,12]$. The utilization of available substrates and the metabolic potential and growth rate of bacteria all play indispensable roles related to their pathogenicity [8]. A previous transcriptomics study demonstrated that nicotinamide adenine dinucleotide (NADH)-dependent flavin oxidoreductase (NFOR) was overrepresented in Mhp pathogenic strain 7448 but not in M. flocculare [13]. In addition, previous comparative proteomics reports demonstrated that there are significant differences in the expression of NFOR between high-and low-virulence Mhp strains [14, 15]. NFOR is an oxidoreductase, the largest class of enzymes, and its function is to catalyze the oxidation of $\mathrm{NADH}$ to $\mathrm{NAD}+$ by reducing molecular $\mathrm{O} 2$ to $\mathrm{H} 2 \mathrm{O}$ or $\mathrm{H} 2 \mathrm{O} 2$ at the same time. Some organisms, such as Nomuraea rileyi, contain these kinds of enzymes, which play important roles in regulating cellular redox and osmotic pressure balance to maintain normal cell growth and development [16]. Therefore, we hypothesized that Mhp NFOR, in addition to its effect on metabolic processes, may have a certain relationship with Mhp virulence.

As a new candidate virulence factor discovered in this study, NFOR was located at the surface of Mhp, as determined by electron microscopy observation and flow cytometry analysis. NFOR could adhere to immortalized porcine bronchial epithelial cells (hTERT-PBECs) and recognize host fibronectin and plasminogen, with higher affinity for fibronectin. It could also induce host cytotoxicity, oxidative stress damage, and apoptosis. Our findings support the notion that NFOR may be a potential novel virulence factor of Mhp, which will provide new ideas and theoretical support for studying the pathogenic mechanisms of Mhp and other mycoplasmas.

\section{Materials And Methods}

\section{Mhp strains and growth conditions}

All five Mhp strains were thawed from frozen Mhp bacterial stocks and subcultured for three generations before use for subsequent analysis. The strains were cultured in modified Friis medium, designated KM2 cell-free medium, which contained $20 \%(\mathrm{v} / \mathrm{v})$ pig serum (sterilized by irradiation from a clean snatchfarrowed porcine colostrum-deprived piglet and produced in our lab) cultivated in a humidified incubator at $37^{\circ} \mathrm{C}$ [17]. Mhp strain 168 was isolated and cultured from a pig exhibiting representative characteristics of mycoplasma pneumonia of swine (MPS) in China[18]. This field strain was gradually attenuated 
through continuous passage to the $380^{\text {th }}$ generation, resulting in the low-virulence strain 168L [19]; the Mhp 168L strain used in this study was passage 353 . Strain JS is a virulent strain that can induce typical characteristics of MPS with a lung lesion score of approximately 15, as mentioned previously [17]. Strain LH is a virulent clinical strain that was isolated in our lab. Strain J (ATCC 25934) was passaged once from the ATCC stock to yield frozen stocks. The titers of Mhp strains were quantified using the $50 \%$ color change unit $\left(\mathrm{CCU}_{50}\right)$ assay [20], which was modified from the CCU assay [21] and tested by quantitative PCR.

\section{RNA transcriptional analysis}

The five Mhp strains were cultured in the abovementioned culture conditions at $37^{\circ} \mathrm{C}$ for $48 \mathrm{~h}$. Then, total RNA was extracted using a Total RNA Extraction Kit (Cat No. R6834, Omega Biotek, Guangzhou, China). HiScript® II Q RT SuperMix for qPCR (+gDNA wiper) (Cat No. R223-01, Vazyme, Nanjing, China) was used to reverse transcribe at least $1 \mu \mathrm{g}$ of total RNA in a $10-\mu \mathrm{L}$ reaction volume before performing qRT-PCR on an ABI 7500 Real Time PCR System with the help of a HiScript ${ }^{\circledR}$ II One Step qRT-PCR SYBR® Green Kit (Cat No. Q221-01, Vazyme, Nanjing, China). qRT-PCR was run using cDNA for the NFOR gene under specific conditions. The P46 gene of Mhp was selected as the internal control. The PCR primers used in the quantitative assays are listed in Table 1. The fold changes in mRNA expression of the NFOR genes of various Mhp strains differing in virulence were determined using the $2^{-}$ $\triangle \triangle C T$ method as described previously [22].

\section{Multiple sequence alignment}

Seventeen amino acid sequences of the NFOR protein from Mhp were retrieved from the National Center for Biotechnology Information and UniProt protein databases, and their homologies were analyzed. All sequences were aligned with the CLUSTAL W program. Phylogenetic inference according to the neighborjoining criterion was performed through Molecular Evolutionary Genetics Analysis version 10 (MEGA 10). A total of 2000 nonparametric bootstrap analyses were used to test the robustness of the hypothesis.

\section{Protein expression and preparation of polyclonal antibody}

The Mhp NFOR gene (MHP168_RS01740) was synthesized by GenScript Biotech Corp. (Nanjing, China) and was expressed in the BL21(DE3) E. coli strain through the pET32a vector, purified using High Affinity Ni-Charged Resin FF affinity columns (Cat No. L00666, GenScript, Nanjing, China), and identified by Western blot analysis. Purified proteins were determined with the BCA Protein Assay Kit (Cat No. P0012S, Beyotime, Shanghai, China), and the protein concentration was calculated before storage at $-70^{\circ} \mathrm{C}$. Polyclonal antibodies against Mhp NFOR were obtained by subcutaneously immunizing 1-month-old New Zealand white rabbits with $1.2 \mathrm{mg}$ of recombinant protein (rNFOR) emulsified in Freund's complete adjuvant for the first immunization (Cat No. F5881, Sigma-Aldrich, St Louis, MO, USA). Each rabbit was then immunized with $1.2 \mathrm{mg}$ of rNFOR emulsified in Freund's incomplete adjuvant (Cat No. F5506, Sigma- 
Aldrich, St Louis, MO, USA) twice at 2-week intervals. Booster immunization was performed once a week after three immunizations before sera were collected.

\section{Enzymatic activity assays}

The enzymatic activity of purified rNFOR was determined by calculating the oxidation of NADH to NAD ${ }^{+}$ at $25^{\circ} \mathrm{C}$. In brief, $5 \mu \mathrm{g} / \mathrm{mL}$ rNFOR, $0.1 \mathrm{M}$ potassium phosphate buffer $(\mathrm{pH} 7.5$, containing $1 \mathrm{mM}$ dithiothreitol), $10 \mu \mathrm{M}$ flavin mononucleotide (FMN) (Cat No. F107158, Aladdin, Shanghai, China) and 0.5 mM NADH (Cat No. N106933, Aladdin, Shanghai, China) were used in this study. The reaction system was $2 \mathrm{~mL}$, and the purified rNFOR was preincubated with FMN for 5 min before NADH was incubated. The optical density (OD) was measured at $340 \mathrm{~nm}$ (OD340). The specific activity was calculated by the following equation:

$$
\mathrm{U} / \mathrm{mg}=\frac{\Delta \mathrm{OD}}{t \times \varepsilon \times l \times m} \times V \times 10^{6}
$$

\section{Surfaceexposed NFOR detection by flow cytometry}

Flow cytometry analysis was used to test whether NFOR is located on the surface of Mhp strains and to probe NFOR surface distributions between the high-virulence strain 168 and the low-virulence strain 168L. Mhp strains 168 and $168 \mathrm{~L}$ (titers of which were $1 \times 10^{8} \mathrm{CCU} / \mathrm{mL}$ ) were incubated with anti-rNFOR serum or with preimmune serum (negative control) at a 1:100 dilution, as reported previously $[23,24]$. Fluorescein isothiocyanate (FITC)-conjugated anti-IgG (Cat No. BA1105, Boster, Wuhan, China) was then used to stain the above Mhp strains, and a BD Accuri C6 flow cytometer was used to measure the fluorescence intensity.

\section{Surface exposed NFOR detection by immunoelectron microscopy}

Mhp strains were cultured and grown to mid-log phase at $10000 \times g$ by centrifugation at $10^{\circ} \mathrm{C}$ for 20 min before each Mhp bacterium was harvested. A total of $1 \times 10^{8} \mathrm{CCU}$ of bacterial suspension was washed three times and finally resuspended in a volume of $50 \mu \mathrm{L}$ of $0.1 \mathrm{M}$ phosphate-buffered saline (PBS, pH 7.4). Immunoelectron microscopy was performed according to previous studies $[25,26]$ with some modifications. Briefly, $5 \mu \mathrm{L}$ of the sample was added to a 400 mesh formvar-coated nickel grid and allowed to stand for $5 \mathrm{~min}$. Then, the grid was fixed with 2\% paraformaldehyde in PBS for 5 min at room temperature (RT) followed by blocking with $1 \%$ negative rabbit serum and blocking buffer (1\% (w/v) BSA in PBS) for $1 \mathrm{~h}$. The samples were then incubated with anti-rNFOR antibody or preimmune serum (negative control) at a 1:10 dilution in blocking buffer for another $1 \mathrm{~h}$ (PBS as a blank control). After washing five times in blocking buffer, the samples were incubated with a secondary gold-conjugated antibody (goat anti-rabbit lgG, 10 nm-gold particles, Cat No. GA1014, Boster, Wuhan, China) at a dilution of 1:20 for 1 more hour. The samples were washed 5 times with PBS for 5 min each time before being fixed in $2 \%$ paraformaldehyde in PBS for $5 \mathrm{~min}$. Then, the grids were washed eight times in distilled water 
and stained with $1 \%$ phosphotungstic acid $(\mathrm{pH} 6.5)$ for $15 \mathrm{~s}$. After the samples were dried by an infrared lamp, they were observed under a Tecnai high-field transmission electron microscope.

\section{Indirect immunofluorescence assay (IFA)}

Immortalized porcine bronchial epithelial cells (hTERT-PBECs) were established and cultured to a density of $80 \%$ in 24-well cell plates with Dulbecco's modified Eagle's medium:nutrient mixture F-12 (DMEM/F12) medium plus 2\% (v/v) fetal bovine serum (Gibco, Grand Island, NY, USA) supplemented with growth factors (Cat No. CC-4175, Lonza, Basel, Switzerland), as we previously made and reported [22]. Cells were washed three times with cold PBS before being fixed with $4 \%$ paraformaldehyde for 10 min at RT. Subsequently, $0.2 \%$ Triton X-100 was used at RT for 3 min, followed by blocking for $2 \mathrm{~h}$ using $3 \%(\mathrm{w} / \mathrm{v})$ BSA in PBS. Cells were incubated with $100 \mu \mathrm{g}$ of purified rNFOR for $1 \mathrm{~h}$ at $37^{\circ} \mathrm{C}$ in a cell incubator before they were washed three times with PBS and incubated with anti-rNFOR antibody at a 1:250 dilution for another $2 \mathrm{~h}$ at $37^{\circ} \mathrm{C}$. After three washes with PBS, the cells were then incubated with a 1:100 dilution of tetraethyl rhodamine isothiocyanate (TRITC)-conjugated anti-IgG (Cat No. SA000072, Proteintech, Rosemont, IL, USA) for $1 \mathrm{~h}$ in a $37^{\circ} \mathrm{C}$ incubator. 6-Diamidino-2-phenylindole (DAPI, Cat No. D8417, Sigma-Aldrich, St Louis, MO, USA) was used for nuclear staining before the cells were observed using a fluorescence microscope (Zeiss, Tokyo, Japan). Instead of rNFOR, BSA was selected as a negative control.

\section{Antibody-mediated adhesion inhibition}

Mhp strains (high-virulence 168 and low-virulence 168L, the titers of which were $1 \times 10^{7} \mathrm{CCU} / \mathrm{mL}$ ) were collected by centrifugation at $10000 \times g$ for 20 min at $10^{\circ} \mathrm{C}$ and resuspended in $500 \mu \mathrm{L}$ of PBS after washing three times with PBS. The samples were preincubated with polyclonal antibody against rNFOR or preimmune serum at a 1:20 dilution for $30 \mathrm{~min}$ in a $37^{\circ} \mathrm{C}$ incubator. Mhp bacteria were suspended in DMEM/F12 before being added to confluent hTERT-PBECs seeded in 24-well cell plates. Plates were then centrifuged at $1000 \times g$ for $10 \mathrm{~min}$ before being placed at $4^{\circ} \mathrm{C}$ for $2 \mathrm{~h}$. After washing three times with PBS, hTERT-PBECs were collected after digestion with $0.125 \%$ trypsin (twice diluted with Hanks medium with $0.25 \%$ trypsin, Cat No. 25200072, Gibco, Grand Island, NY, USA), and the cells were centrifuged at $1300 \mathrm{rpm}$ for $10 \mathrm{~min}$ after adding DMEM/F12 containing 10\% FBS to stop cell digestion. Following Mhp bacterial genome extraction, quantitative real-time PCR was then performed as previously reported [27]; the real-time PCR primers are shown in Table 1. Experiments were performed in triplicate, and data were analyzed using SPSS 20.0. Mhp titers were quantified using the $\mathrm{CCU}_{50}$ assay mentioned above.

\section{Surface plasmon resonance (SPR) analysis}

SPR analysis was performed according to our previous study [26] using a Biacore X100 Plus instrument (GE Healthcare, Boston, MA, USA). Fibronectin and plasminogen were diluted to $50 \mu \mathrm{g} / \mathrm{mL}$ before they were linked covalently to the CM5 sensor chip as a ligand using an amine coupling kit (Biacore AB, Cytiva, Guangzhou, China). The immobilization of soluble fibronectin and plasminogen produced approximately 
2000 resonance units (RUs). The binding kinetics were measured by increasing the concentration (0-4000 $\mathrm{nmol} / \mathrm{L}$ ) of the analyte (Mhp NFOR) in running buffer (HBS-EP), which consisted of $10 \mathrm{mM} \mathrm{HEPES}, 150$ $\mathrm{mM} \mathrm{NaCl}, 3 \mathrm{mM}$ EDTA and 0.05\% (v/v) surfactant P20 (Biacore AB, Cytiva, Guangzhou, China), with a flow rate of $30 \mu \mathrm{L} / \mathrm{min}$, passing through immobilized Mhp NFOR at $20^{\circ} \mathrm{C}$ for $3 \mathrm{~min}$. The dissociation phase was monitored for 1000 seconds by allowing buffer to flow through the chip. Biacore X100 control software was used to manually analyze the binding kinetics.

\section{Far-Western blot (Far-WB) analysis}

Fifteen micrograms of rNFOR was resolved by $10 \%$ SDS-PAGE and then transferred to a PVDF membrane (Cat No. IPFL00010, Millipore, Darmstadt, Germany). After three washes with PBS, the membrane was blocked with $5 \%$ skimmed milk in TBST (TBS containing $0.5 \%$ Tween 20), which served as the blocking buffer, before being placed in a $37^{\circ} \mathrm{C}$ incubator for $2 \mathrm{~h}$ with gentle shaking. Then, the membrane was incubated with $15 \mu \mathrm{g} / \mathrm{mL}$ fibronectin (Cat No. F1056, Sigma-Aldrich, Darmstadt, Germany) or plasminogen (Cat No. SRP6518, Sigma-Aldrich, Darmstadt, Germany) at $37^{\circ} \mathrm{C}$ for another 2 h. After another three washes with TBST, membranes were subsequently incubated with anti-fibronectin antibody (Cat No. ab299, Abcam, Cambridge, UK) at a 1:1000 dilution or anti-plasminogen antibody (Cat No. ABP55618, Annkine, Wuhan, China) at a 1:400 dilution in blocking buffer for $2 \mathrm{~h}$ in a $37^{\circ} \mathrm{C}$ incubator. The membranes were then incubated with the secondary antibody (HRP-conjugated goat antirabbit lgG) (Cat No. BA1055, Boster, Wuhan, China) at a 1:2000 dilution for $2 \mathrm{~h}$ in a $37^{\circ} \mathrm{C}$ incubator after three washes with TBST. Finally, the membranes were developed with Electro-Chemi-Luminescence (ECL) substrate using a ChemiDoc XRS+ system (Bio-Rad). Instead of rNFOR, BSA was used as a negative control.

\section{Quantification of lactate dehydrogenase (LDH) release}

hTERT-PBECs were seeded in 24-well cell plates one night before cell growth reached $80 \%$ confluence. First, the cells were incubated with purified rNFOR protein at different concentrations ( $5 \mu \mathrm{g}, 10$ $\mu \mathrm{g}, 15 \mu \mathrm{g}, 20 \mu \mathrm{g})$. Six hours later, culture supernatants were collected, and LDH activity was measured with a CytoTox $96^{\circledR}$ Non-Radioactive Cytotoxicity Assay (Cat No. G1780, Promega, Madison, WI, USA) following the manufacturer's instructions. The corrected values in the formula below were used to calculate the percentage of cytotoxicity: percent cytotoxicity $=100 \times$ experimental LDH release $\left(\mathrm{OD}_{490}\right)$ /maximum LDH release $\left(\mathrm{OD}_{490}\right)$. PBS was used instead of $\mathrm{rNFOR}$ as a negative control, and three Mhp strains (strains JS and $\mathrm{J}$ and $168 \mathrm{~L}, 1 \times 10^{8} \mathrm{CCU} / \mathrm{mL}$ ) that differed in virulence were used as positive controls.

\section{Reactive oxygen species (ROS) detection}

hTERT-PBECs were seeded in 24-well plates one day before cell growth reached $80 \%$ confluence. Cells were incubated with $20 \mu \mathrm{g}$ of purified rNFOR in a $37^{\circ} \mathrm{C}$ incubator for $6 \mathrm{~h}$. ROS detection was measured using a ROS-Glo ${ }^{T M} \mathrm{H}_{2} \mathrm{O}_{2}$ Assay (Cat No. G8820, Promega, Madison, WI, USA), and relative luminescence 
units (RLU) were recorded using a plate reader. PBS was used as a negative control, and positive controls were the RLUs from three Mhp strains (strain JS, J and $168 \mathrm{~L}, 1 \times 10^{8} \mathrm{CCU} / \mathrm{mL}$ ).

\section{Apoptosis assay}

hTERT-PBECs were seeded one night before at $2 \times 10^{5}$ cells/well in culture medium of a total volume of $500 \mu \mathrm{L}$ in 24-well cell plates. hTERT-PBECs were then incubated with $20 \mu \mathrm{g}$ of purified rNFOR at $37^{\circ} \mathrm{C}$ for $12 \mathrm{~h}$, with cells grown from culture medium DMEM/F12 plus 2\% FBS and epithelial growth factors to maintain the DMEM/F12 without adding the above reagents as a negative control, and Mhp strains that differed in virulence (high-virulence strain JS and low-virulence strain $168 \mathrm{~L}, 1 \times 10^{8} \mathrm{CCU} / \mathrm{mL}$ ) were used as positive controls. To explore whether antiserum to rNFOR could block and reduce the apoptosis induced by Mhp in hTERT-PBECs, we preincubated Mhp strains (JS and 168L, $1 \times 10^{8} \mathrm{CCU} / \mathrm{mL}$ ) with rabbit polyclonal antibody raised against rNFOR at a $1: 20$ dilution at $37^{\circ} \mathrm{C}$ for half an hour before they were applied to hTERT-PBECs seeded in 24-well plates in a $37^{\circ} \mathrm{C}$ incubator for $12 \mathrm{~h}$. The apoptosis rate was detected and calculated using a dual apoptosis detection kit (Cat No. A211, Vazyme, Nanjing, China) with Annexin V-FITC/PI.

\section{Statistical analysis}

Data for the adhesion rate from quantitative real-time PCR analysis and the apoptosis rate were analyzed by GraphPad Prism 6 software and FlowJo software v7.6. Relative NFOR mRNA expression levels between the moderate-virulence strain $\mathrm{J}$ or the low-virulence strain $168 \mathrm{~L}$ and the high-virulence Mhp strains 168, JS, and LH were assessed via multiple comparisons of analysis of variance (ANOVA). The adhesion rates between anti-NFOR serum or negative serum and groups of the high-virulence strain 168 or between low-virulence strain $168 \mathrm{~L}$ and anti-NFOR serum or negative serum were compared by the multiple $t$ test. $P<0.05$ was considered a significant difference, and $P<0.01$ was considered an extremely significant difference.

\section{Results}

\section{Significant differences in NFOR transcription levels between high- and low-virulence Mhp strains}

The relative quantitative RT-PCR results showed that there indeed existed significant differences in NFOR transcription levels among the five Mhp strains that differed in virulence. Figure 1 shows the mRNA expression levels of the NFOR gene in five Mhp strains, with the Mhp strains $168 \mathrm{~L}$ (Fig. 1A) and $\mathrm{J}$ (Fig. 1B) used as controls. The mRNA expression levels of the NFOR gene were significantly upregulated in the high-virulence Mhp strains 168, LH and JS compared to the low-virulence Mhp strain 168L. The mRNA expression levels of the NFOR gene in Mhp strain $\mathrm{J}$ remained unchanged, as the expression levels were far less than a 2-fold change (Fig. 1A), which is usually regarded as the criterion for distinguishing whether expression is significantly different [28]. Similarly, the mRNA expression levels of the NFOR gene were significantly upregulated in the high-virulence Mhp strains 168, LH and JS compared to the moderate-virulence Mhp strain J (Fig. 1B). In contrast, the mRNA expression levels of the NFOR gene 
in Mhp strain 168L were decreased compared with those of strain $\mathrm{J}$, but the difference was not significant.

\section{Bioinformatics analysis, protein expression and enzymatic activity of Mhp NFOR}

Seventeen protein sequences of NFOR from Mhp were retrieved from the NCBI and UniProt databases, and the homologies between them were analyzed. As shown in Fig. 2A, all protein sequences were aligned with the CLUSTAL W program (Fig. 2A). Molecular Evolutionary Genetics Analysis version 10 (MEGA 10) was used to make phylogenetic inferences based on the neighbor-joining criterion. The robustness of the hypothesis was tested through 2000 nonparametric bootstrap analyses (Fig. 2B). It was found that all Mhp NFOR proteins had only minimal differences in amino acid positions from the results of multiple sequence alignments. Similarly, evolutionary analysis revealed that the overall homology of NFOR among all Mhp strains reached approximately $96.92 \%$, suggesting that NFOR is a relatively highly conserved protein and has little effect among different Mhp strains.

The full-length coding sequence (CDS) of Mhp 168 strain NFOR is $1173 \mathrm{bp}$, with the predicted protein containing 390 amino acids. On the basis of codon optimization, a prokaryotic expression vector pET-32a-NFOR was constructed, and it was expressed in Escherichia coli BL21 (DE3) to study the enzyme activity of rNFOR after purification. The enzymatic specific activity of the rNFOR protein was determined to be $29.12 \mathrm{lU} / \mathrm{mg}$.

\section{NADH-dependent flavin oxidoreductase is located at the surface of Mhp cells}

To confirm whether NFOR is located on the surface of Mhp, two tests were performed. Flow cytometry analysis revealed that the outer membrane-localized NFOR can approach the NFOR-specific antibody through the surface in both Mhp strains 168 and 168L, indicating that the NFOR antigen is present on the cell surface of the Mhp strain. There was no significant difference in the mean fluorescence intensity (MFI) between Mhp strain 168L incubated with preimmune serum and $168 \mathrm{~L}$ treated with antirecombinant protein NFOR (anti-rNFOR) serum (Fig. 3A), while the MFI of strain 168 treated with preimmune serum was approximately 4-fold lower than that of Mhp 168 treated with anti-rNFOR serum (Fig. 3B). In addition, Mhp cells bind antibodies against the Mhp NFOR protein surrounding their surface, as revealed by immune electron microscopy. The antibodies were localized and restricted to the peripheral area of Mhp cells in both the Mhp strain JS and strain 168, while in the preimmune serumtreated negative group, gold particles were not visible (Fig. 3C).

\section{rNFOR adheres to hTERT-PBECs, and treatment with anti-rNFOR serum inhibits adherence to Mhp}

An indirect immunofluorescence assay (IFA) was used to determine whether Mhp NFOR could specifically adhere to the surface of hTERT-PBECs. The results revealed obvious orange-red punctate fluorescence on the surface of immortalized hTERT-PBECs incubated with rNFOR, whereas in the negative control group, no specific fluorescence was found around cell nuclei stained with DAPI (Fig. 4A), suggesting that rNFOR could specifically bind to hTERT-PBECs. 
To further assess the role of NFOR in Mhp adhesion to hTERT-PBECs, an antibody inhibition assay was conducted. Anti-rNFOR serum decreased the adherence of Mhp (both strains 168 and 168L) to hTERT-PBECs compared to that of the control group, which was treated with negative serum. Compared with the adhesion of Mhp in the absence of anti-rNFOR polyclonal antibody, the adhesion level was shown as the adhesion rate. Mhp strains incubated with anti-rNFOR antibody led to $48.7 \%$ (strain 168) and $51.75 \%$ (strain 168L) $(p<0.001)$ reductions in the adhesion rate of Mhp to hTERT-PBECs (Fig. 4B). The results further indicated that NFOR of Mhp plays an irreplaceable role in the adherence of Mhp to host cells.

\section{Identification of rNFOR binding ligands}

To determine whether the components of hTERT-PBECs interact with NFOR, surface plasmon resonance (SPR) and far-Western blot analysis were used to examine the interactions between NFOR of Mhp and fibronectin and plasminogen. Mhp NFOR could bind to fibronectin and plasminogen in a dose-dependent manner, with equilibrium dissociation constant (KD) values of $233.3 \mathrm{nmol}$ and $394.8 \mathrm{nmol}$, respectively (Fig. 5A), indicating that Mhp NFOR could specifically bind to both fibronectin and plasminogen and had a relatively high affinity for fibronectin.

Expression of purified recombinant protein rNFOR in the pET-32a expression vector is shown in panel "a" of Fig. 5B, with a recombinant protein rNFOR size of $44 \mathrm{kDa}$. The expression of rNFOR was detected using a polyclonal antibody in a Western blotting experiment. As shown in panel "b" of Fig. 5B, a clear band appeared at position $44 \mathrm{kDa}$ regardless of whether it was from the whole bacterial protein or the purified protein rNFOR. As shown in Fig. 5C, before the final reaction with fibronectin or plasminogen, the corresponding band was observed in the reaction of rNFOR with anti-rNFOR antibody (positive control), but no specific reaction was observed in the negative control (which used BSA was instead of anti-rNFOR antibody). The results showed that Mhp NFOR has a strong affinity for plasminogen and fibronectin and that the binding ability of NFOR and fibrinogen is better than that of the Mhp and plasminogen.

\section{rNFOR induced cytotoxicity, oxidative stress damage and apoptosis of hTERT-PBECs}

The cytotoxicity of rNFOR on hTERT-PBECs was determined by the CytoTox $96^{\circledR}$ Non-Radioactive Cytotoxicity test, and it was found that the viability of cells was significantly reduced by rNFOR in a dosedependent manner (Fig. 6A). In addition, rNFOR induced oxidative stress damage in hTERT-PBECs (Fig. $6 \mathrm{~B})$. The results also demonstrated that the cytotoxicity and host cell cellular oxidative stress of Mhp against hTERT-PBECs were significantly correlated with the differing virulence of pathogenic Mhp strains (Fig. 6A and 6B).

The flow cytometry results further indicated that rNFOR could induce apoptosis in hTERT-PBECs (Fig. 7A), and the late-stage apoptosis (upper right quadrant) rate was upregulated by $82.4 \%$ compared with the negative control group (4.9\%). The late-stage apoptosis induced by Mhp strain JS or 168L in hTERTPTECs was significantly decreased when rNFOR was blocked with an anti-rNFOR antibody. The late-stage apoptotic cell percentage decreased by $41.9 \%$ and $35.8 \%$, respectively (Fig. 7B and 7C). 


\section{Discussion}

To date, all mycoplasmas cultured and identified are known to be host parasites, the hosts of which include humans or animals, with a higher degree of specificity to hosts and tissues [29]. The main habitats of mycoplasmas are respiratory and genitourinary tracts, serous membranes and epithelial surfaces of mammary glands of certain animal species. Mhp is a host-specific pathogen that infects only pigs. Currently, many virulence factors of Mhp have been revealed, and their roles include adhesion, invasion, and intracellular proliferation; however, the molecular mechanisms of infection and pathogenesis have not been fully elucidated [9]. In recent years, some studies have indicated that several metabolic enzymes play an important role in host-pathogen interactions during Mycoplasma infections[8, $23,26]$. "Omics" studies have shown that there are significant differences in the expression of NFOR between high-virulence Mhp strain 7448 and moderate-virulence strain $\mathrm{J}$ and nonpathogenic $M$. flocculare through transcriptomics comparison [13] or between high- and low-virulence Mhp strains through proteomics comparison [14, 15]; however, a previous study classified NFOR as a "nonvirulent" protein [14] in the supplementary file, but with no verification.

However, here, the mRNA expression levels of the NFOR gene in pathogenic high-virulence Mhp strains 168, LH and JS were significantly upregulated compared with either moderate-virulence strain J or lowvirulence strain 168L. This result preliminarily confirmed that NFOR was correlated with the virulence of Mhp. Further investigation showed that the NFOR antigen was located on the cell surface of Mhp by both $\mathrm{MFI}$ analysis through flow cytometry and immunoelectron microscopy observation. Compared with the nonpathogenic $168 \mathrm{~L}$ strain, the fluorescence intensity of the pathogenic high-virulence strain 168 was increased by approximately 4 -fold, further indicating that NFOR may be responsible for the virulence of Mhp.

The adhesion of all microorganisms, including mycoplasma, to their host cells is a key step for their colonization and subsequent infection of the host. Adhesion ability is an important factor that reflects bacterial virulence [30]. Mhp is mainly found on the mucosal surface along the entire swine respiratory tract, including the trachea, bronchi and bronchioles, inducing ciliostasis and loss of cilia [31]. The first stage of pathogenesis is the adhesion of Mhp to cilia in epithelial cells of the respiratory mucosa by means of adhesins. Here, we found that NFOR could adhere to the immortalized porcine bronchial epithelial cells (hTERT-PBECs) established in our previous study [22]. In addition, preneutralizing Mhp with polyclonal antiserum to rNFOR obviously decreased the adherence of Mhp to host cells.

Fibronectin has been studied more extensively, and a popular extracellular matrix protein has been identified that forms a molecular bridge between pathogens and host cell receptors [32, 33]. According to our previous studies $[23,26]$, several fibronectin-binding bacterial proteins have been found to mediate the adhesion of bacteria to host cells and subsequent invasion by binding to fibronectin. Therefore, fibronectin binding protein plays a critical role in the pathogenic process of bacteria, including mycoplasmas. In addition to fibronectin, a variety of bacteria can sequester the host zymogen plasminogen to the cell surface. Once localized to the bacterial surface, plasminogen can act as a 
cofactor for adhesion or, after being activated as plasmin, can provide an effective source of proteolytic activity. The recruitment of plasminogen to the surface of bacterial cells is directly mediated by specialized cell surface receptors or cytoplasmic and glycolytic pathway proteins located on the surface of bacterial cells or is indirectly mediated through interactions with host plasma proteins such as fibrinogen [34]. In this study, we found that rNFOR could specifically bind to fibronectin and plasminogen and had a higher affinity for fibronectin. It is possible that fibronectin might function as the main receptor of NFOR and that plasminogen acts as a cofactor to mediate the adhesion process of Mhp. Further characterization of the interaction between fibronectin and plasminogen would be useful and helpful in improving the understanding of the adhesion-related factors of Mhp.

Lactate dehydrogenase (LDH) is a stable cytoplasmic enzyme that converts lactate to pyruvate. LDH release assays of damaged cells are a method to detect cell viability from the perspective of cell membrane integrity [35]. Our results showed that NFOR could induce cytotoxicity in hETRT-PBECs in a dose-dependent pattern, and the LDH release of NFOR in host cells was positively correlated with various Mhp strains that differed in virulence. ROS are constantly generated during numerous cellular processes, one of the main sources of which is aerobic respiration, but most are often offset by antioxidant proteins. In addition, a large amount of ROS is produced from bacterial or viral infections, inflammatory reactions, ionizing radiation, and many chemical drugs [36-38]. In these processes, if the induced ROS is more than the upper limit offset by antioxidant proteins, so-called "oxidative stress" will be generated. Unlike cell necrosis, apoptosis is not a passive process but a phenomenon that induces autologous injury through a series of signal activation, protein expression and regulation processes [39]. A recent study demonstrated that ROS deposition is thought to be a direct cause of apoptosis because ROS induce strong cytotoxicity to host cells. Thus, when the intracellular ROS content shows a large increase, it will stimulate oxidative stress and induce apoptosis [40-42].

In this study, we found that rNFOR could induce oxidative stress damage in immortalized porcine bronchial epithelial cells (hTERT-PBECs) in Mhp strains that differed in virulence. Moreover, the $\mathrm{H}_{2} \mathrm{O}_{2}$ release induced against cells by the low-virulence Mhp strain 168L was significantly lower than that induced by one high-virulence strain and one moderate-virulence strain (JS and J). Flow cytometry results further indicated that rNFOR could also induce apoptosis in hTERT-PBECs, and the late-stage apoptosis rate was upregulated by $82.4 \%$ compared with the negative control group $(4.9 \%)$ after changing the medium to maintenance medium without FBS and growth factors after $12 \mathrm{~h}$. The late-stage apoptosis induced by either Mhp from the high-virulence strain JS or the low-virulence strain 168L in hTERT-PBECs was significantly decreased when rNFOR was blocked with anti-rNFOR polyclonal antibody, with latestage apoptotic cell percentages decreasing by $41.9 \%$ and $35.8 \%$, respectively. In conclusion, these results suggested that NFOR, functioning as more than only a metabolic enzyme, may act as a potential new virulence factor of Mhp, which will provide certain theoretical support and new ideas for the research and development of live-attenuated or subunit vaccines against Mhp.

\section{Conclusions}


In this study, in addition to being a metabolic enzyme related to oxidative stress, NFOR may be a potential novel virulence factor of Mhp, thus contributing to the pathogenesis process of Mhp, providing new ideas and theoretical support for studying the pathogenic mechanisms of other mycoplasmas.

\section{Abbreviations}

Mhp: Mycoplasma hyopneumoniae; NFOR: Nicotinamide adenine dinucleotide dependent flavin oxidoreductase; hTERT-PBECs: immortalized porcine bronchial epithelial cells; FMN: flavin mononucleotide; IFA: indirect immunofluorescence assays; SPR: surface plasmon resonance; Far-WB: Far-Western blot; LDH: lactate dehydrogenase; ROS: Reactive oxygen species.

\section{Declarations}

\section{Ethics Statement and consent to participate}

All animal experiments of polyclonal antibodies obtained from rabbits were approved by the Committee on the Ethics of Animal Experiments and performed in Jiangsu Academy of Agricultural Sciences, with the approve number is SYXK (Su) 2015-0019 and SYXK (Su)2020-0023). Animal experiment including sample collection were in strict accordance with the guidelines of Jiangsu Province Animal Regulations (Government Decree No. 45).

\section{Consent for publication}

Not applicable.

\section{Data availability}

All data and materials are included in the manuscript and will be available from the corresponding author on reasonable request.

\section{Competing interests}

The authors declare that they have no competing interests.

\section{Funding}

This work was supported by National Natural Science Foundation of China (31800161, 31800160, 31900159, 31770193); Natural Science Foundation of Jiangsu Province (BK20180297); the Independent Research Project Program of Jiangsu Key Laboratory for Food Quality and Safety-State Key Laboratory Cultivation Base, Ministry of Science and Technology, China (2019sy004); Jiangsu Agriculture Science and Technology Innovation Fund (CX(20)1006).

\section{Authors' contributions}


$X X$ carried out most of the experiments described in the manuscript and wrote the article; FH, RC, JJW and $\mathrm{JL}$ helped preparing the recombinant protein and preparing the rabbit hyperimmune sera; YNW cultured Mhp strains; HYW and ZZZ helped doing relevant in vivo cell cytotoxicity, cell apoptosis experiment; YB performed real-time PCR experiment; and QYX and GQS helped to revise the manuscript; ZXF conceived the study and contributed in its design and coordination. All authors read and approved the final manuscript.

\section{Acknowlegement}

Thanks to Jin Wang of Nanjing Agricultural University for the assistance in performing cell apoptpsis analysis. Thanks for the editing of our manuscript by an English professional with a scientific background at American Journal Experts, which could be verified at www.aje.com/certificate (certificate verification key: 4AD3-F20B-C8DC-03E3-5D32).

\section{References}

1. Razin, S.; Yogev, D.; Naot, Y., Molecular biology and pathogenicity of mycoplasmas. Microbiol Mol Biol Rev. 1998; 62, (4), 1094-156.

2. Peterson, S. N.; Fraser, C. M., The complexity of simplicity. Genome Biol. 2001;2, (2), COMMENT2002.

3. Chopra-Dewasthaly, R.; Baumgartner, M.; Gamper, E.; Innerebner, C.; Zimmermann, M.; Schilcher, F.; Tichy, A.; Winter, P.; Jechlinger, W.; Rosengarten, R.; Spergser, J., Role of Vpma phase variation in Mycoplasma agalactiae pathogenesis. FEMS Immunol Med Mic. 2012; 66, (3), 307-22.

4. Burki, S.; Gaschen, V.; Stoffel, M. H.; Stojiljkovic, A.; Frey, J.; Kuehni-Boghenbor, K.; Pilo, P., Invasion and persistence of Mycoplasma bovis in embryonic calf turbinate cells. Vet Res. 2015; 46, 53.

5. Hegde, S.; Hegde, S.; Spergser, J.; Brunthaler, R.; Rosengarten, R.; Chopra-Dewasthaly, R., In vitro and in vivo cell invasion and systemic spreading of Mycoplasma agalactiae in the sheep infection model. Int J Med Microbiol. 2014; 304, (8), 1024-31.

6. Much, P.; Winner, F.; Stipkovits, L.; Rosengarten, R.; Citti, C., Mycoplasma gallisepticum: Influence of cell invasiveness on the outcome of experimental infection in chickens. FEMS Immunol Med Mic. 2002; 34, (3), 181-6.

7. Buim, M. R.; Buzinhani, M.; Yamaguti, M.; Oliveira, R. C.; Mettifogo, E.; Ueno, P. M.; Timenetsky, J.; Santelli, G. M.; Ferreira, A. J., Mycoplasma synoviae cell invasion: elucidation of the Mycoplasma pathogenesis in chicken. Comp. Immunol. Microb. 2011;34, (1), 41-7.

8. Hegde, S.; Rosengarten, R.; Chopra-Dewasthaly, R., Disruption of the pdhB pyruvate dehydrogenase [corrected] gene affects colony morphology, in vitro growth and cell invasiveness of Mycoplasma agalactiae. PloS one. 2015, 10, (3), e0119706.

9. Maes, D.; Sibila, M.; Kuhnert, P.; Segales, J.; Haesebrouck, F.; Pieters, M., Update on Mycoplasma hyopneumoniae infections in pigs: Knowledge gaps for improved disease control. Tranbound Emerg 
Dis. 2018; 65 Suppl 1, 110-124.

10. Leal Zimmer, F. M. A.; Paes, J. A.; Zaha, A.; Ferreira, H. B., Pathogenicity \& virulence of Mycoplasma hyopneumoniae. Virulence. 2020;11, (1), 1600-1622.

11. Pilo, P.; Vilei, E. M.; Peterhans, E.; Bonvin-Klotz, L.; Stoffel, M. H.; Dobbelaere, D.; Frey, J., A metabolic enzyme as a primary virulence factor of Mycoplasma mycoides subsp. mycoides small colony. J Bacteriol. 2005; 187, (19), 6824-31.

12. Rohmer, L.; Hocquet, D.; Miller, S. I., Are pathogenic bacteria just looking for food? Metabolism and microbial pathogenesis. Trends Microbiol. 2011; 19, (7), 341-8.

13. Siqueira, F. M.; Gerber, A. L.; Guedes, R. L.; Almeida, L. G.; Schrank, I. S.; Vasconcelos, A. T.; Zaha, A., Unravelling the transcriptome profile of the swine respiratory tract mycoplasmas. PloS one. 2014; 9 , (10), e110327.

14. Li, S.; Fang, L.; Liu, W.; Song, T.; Zhao, F.; Zhang, R.; Wang, D.; Xiao, S., Quantitative proteomic analyses of a pathogenic strain and its highly passaged attenuated strain of Mycoplasma hyopneumoniae. BioMed Res Int. 2019; 2019, 4165735.

15. Reolon, L. A.; Martello, C. L.; Schrank, I. S.; Ferreira, H. B., Survey of surface proteins from the pathogenic Mycoplasma hyopneumoniae strain 7448 using a biotin cell surface labeling approach. PloS one. 2014; 9, (11), e112596.

16. Liu, J.; Yin, Y.; Song, Z.; Li, Y.; Jiang, S.; Shao, C.; Wang, Z., NADH: flavin oxidoreductase/NADH oxidase and ROS regulate microsclerotium development in Nomuraea rileyi. World J Microb Biot. 2014; 30, (7), 1927-35.

17. Xiong, Q.; Wei, Y.; Feng, Z.; Gan, Y.; Liu, Z.; Liu, M.; Bai, F.; Shao, G., Protective efficacy of a live attenuated Mycoplasma hyopneumoniae vaccine with an ISCOM-matrix adjuvant in pigs. Vet J. 2014; 199, (2), 268-74.

18. Ho, C.; Chu, T.; Chin, H.; Mao, H.; Yeh, A.; Chen, C.; Chang, S.; Chang, D., Microagglutination test for the diagnosis of swine mycoplasmal pneumonia and the identification of Mycoplasmas. Acta Veterinaria Zootechnica Sinica. 1980; 11, (3), 175-186.

19. Liu, W.; Xiao, S.; Li, M.; Guo, S.; Li, S.; Luo, R.; Feng, Z.; Li, B.; Zhou, Z.; Shao, G.; Chen, H.; Fang, L., Comparative genomic analyses of Mycoplasma hyopneumoniae pathogenic 168 strain and its highpassaged attenuated strain. BMC genomics. 2013; 14, 80.

20. Leigh, S. A.; Evans, J. D.; Branton, S. L.; Collier, S. D., The effects of increasing sodium chloride concentration on Mycoplasma gallisepticum vaccine survival in solution. Avian Dis. 2008; 52, (1), 136-8.

21. Furr, P. M.; Taylor-Robinson, D., Factors influencing the ability of different mycoplasmas to colonize the genital tract of hormone-treated female mice. Int J Exp Pathol. 1993; 74, (1), 97-101.

22. Xie, X.; Gan, Y.; Pang, M.; Shao, G.; Zhang, L.; Liu, B.; Xu, Q.; Wang, H.; Feng, Y.; Yu, Y.; Chen, R.; Wu, M.; Zhang, Z.; Hua, L.; Xiong, Q.; Liu, M.; Feng, Z., Establishment and characterization of a telomeraseimmortalized porcine bronchial epithelial cell line. J Cell Physiol. 2018; 233, (12), 9763-9776. 
23. Yu, Y.; Liu, M.; Hua, L.; Qiu, M.; Zhang, W.; Wei, Y.; Gan, Y.; Feng, Z.; Shao, G.; Xiong, Q., Fructose-1,6bisphosphate aldolase encoded by a core gene of Mycoplasma hyopneumoniae contributes to host cell adhesion. Vet Res. 2018; 49, (1), 114.

24. Zhu, W.; Zhang, Q.; Li, J.; Wei, Y.; Cai, C.; Liu, L.; Xu, Z.; Jin, M., Glyceraldehyde-3-phosphate dehydrogenase acts as an adhesin in Erysipelothrix rhusiopathiae adhesion to porcine endothelial cells and as a receptor in recruitment of host fibronectin and plasminogen. Vet Res. 2017; 48, (1), 16.

25. Hegermann, J.; Halbedel, S.; Dumke, R.; Regula, J.; Gabdoulline, R. R.; Mayer, F.; Stulke, J.; Herrmann, R., The acidic, glutamine-rich Mpn474 protein of Mycoplasma pneumoniae is surface exposed and covers the complete cell. Microbiology. 2008; 154, (Pt 4), 1185-1192.

26. Chen, R.; Yu, Y.; Feng, Z.; Gan, R.; Xie, X.; Zhang, Z.; Xie, Q.; Wang, W.; Ran, T.; Zhang, W.; Xiong, Q.; Shao, G., Featured Species-Specific Loops Are Found in the Crystal Structure of Mhp Eno, a Cell Surface Adhesin From Mycoplasma hyopneumoniae. Front Cell Infect Mi. 2019; 9, 209.

27. Wu, Y.; Jin, M.; Bai, F.; Zhang, X.; Hua, L.; Lei, Z.; Shao, G., Development and application of TaqManBHQ real time PCR assay for detection of Mycoplasma hyopneumoniae P97. Chinese Veterinary Science. 2012; 42, (12), 1268-1272.

28. Xie, X.; Pang, M.; Liang, S.; Lin, Y.; Zhao, Y.; Qiu, D.; Liu, J.; Dong, Y.; Liu, Y., Cellular microRNAs influence replication of H3N2 canine influenza virus in infected cells. Vet Microbiol. 2021;257, 109083.

29. Gautier-Bouchardon, A. V., Antimicrobial Resistance in Mycoplasma spp. Microbiol Spectr. 2018; 6, (4).

30. Zhao, G.; Zhang, H.; Chen, X.; Zhu, X.; Guo, Y.; He, C.; Anwar Khan, F.; Chen, Y.; Hu, C.; Chen, H.; Guo, A., Mycoplasma bovis NADH oxidase functions as both a NADH oxidizing and 02 reducing enzyme and an adhesin. Sci Rep-UK. 2017;7, (1), 44.

31. Blanchard, B.; Vena, M. M.; Cavalier, A.; Le Lannic, J.; Gouranton, J.; Kobisch, M., Electron microscopic observation of the respiratory tract of SPF piglets inoculated with Mycoplasma hyopneumoniae. Vet Microbiol. 1992; 30, (4), 329-41.

32. Schwarz-Linek, U.; Hook, M.; Potts, J. R., Fibronectin-binding proteins of gram-positive cocci. Microbes Infect. 2006; 8, (8), 2291-8.

33. Henderson, B.; Nair, S.; Pallas, J.; Williams, M. A., Fibronectin: a multidomain host adhesin targeted by bacterial fibronectin-binding proteins. FEMS Microbiol Rev. 2011; 35, (1), 147-200.

34. Sanderson-Smith, M. L.; De Oliveira, D. M.; Ranson, M.; McArthur, J. D., Bacterial plasminogen receptors: mediators of a multifaceted relationship. J Biomed Biotechnol. 2012; 2012, 272148.

35. Jing, X.; Park, J. H.; Peters, T. M.; Thorne, P. S., Toxicity of copper oxide nanoparticles in lung epithelial cells exposed at the air-liquid interface compared with in vivo assessment. Toxicology in vitro : an international journal published in association with BIBRA. 2015; 29, (3), 502-11.

36. Zhang, Z.; Rong, L.; Li, Y. P., Flaviviridae Viruses and oxidative stress: implications for viral pathogenesis. Oxid Med Cell Longev. 2019; 2019, 1409582. 
37. Grant, S. S.; Hung, D. T., Persistent bacterial infections, antibiotic tolerance, and the oxidative stress response. Virulence. 2013; 4, (4), 273-83.

38. Bai, F.; Ni, B.; Liu, M.; Feng, Z.; Xiong, Q.; Xiao, S.; Shao, G., Mycoplasma hyopneumoniae-derived lipidassociated membrane proteins induce apoptosis in porcine alveolar macrophage via increasing nitric oxide production, oxidative stress, and caspase-3 activation. Vet Immunol Immunop. 2013; 155, (3), $155-61$.

39. Obeng, E., Apoptosis (programmed cell death) and its signals - A review. Brazilian journal of biology $=$ Revista brasleira de biologia. 2021; 81, (4), 1133-1143.

40. Li, Y.; Jiang, Z.; Xue, D.; Deng, G.; Li, M.; Liu, X.; Wang, Y., Mycoplasma ovipneumoniae induces sheep airway epithelial cell apoptosis through an ERK signalling-mediated mitochondria pathway. BMC Microbiol. 2016; 16, (1), 222.

41. Halliwell, B.; Whiteman, M., Measuring reactive species and oxidative damage in vivo and in cell culture: how should you do it and what do the results mean? Brit J Pharmacol.2004; 142, (2), 231-55.

42. Simon, H. U.; Haj-Yehia, A.; Levi-Schaffer, F., Role of reactive oxygen species (ROS) in apoptosis induction. Apoptosis : an international journal on programmed cell death. 2000; 5, (5), 415-8.

\section{Tables}

Table 1 Primers in this study.

\begin{tabular}{|ll|}
\hline Primers name & Summary of Functions or Sequences $\left(5^{\prime}-3^{\prime}\right)$ \\
\hline NFOR-F & GGAACGAAGAGGCGGTAAT \\
\hline NFOR-R & CAGGCACAAATGCTGAAGA \\
\hline Mhp P46-F & TCACTTGCGGCGGGTCTAT \\
\hline Mhp4-R & TTGCTTGTTCGGCCATTCC \\
\hline Mhp183-F & For real-time PCR analysis of NFOR gene expression at the transcriptional level \\
\hline Mhp183-P & ACTGGCTGAACTTCATCTGGGCTA \\
\hline & For real-time PCR analysis of $M h p$ \\
\hline
\end{tabular}

Mhp: Mycoplasma hyopneumoniae

\section{Figures}


A

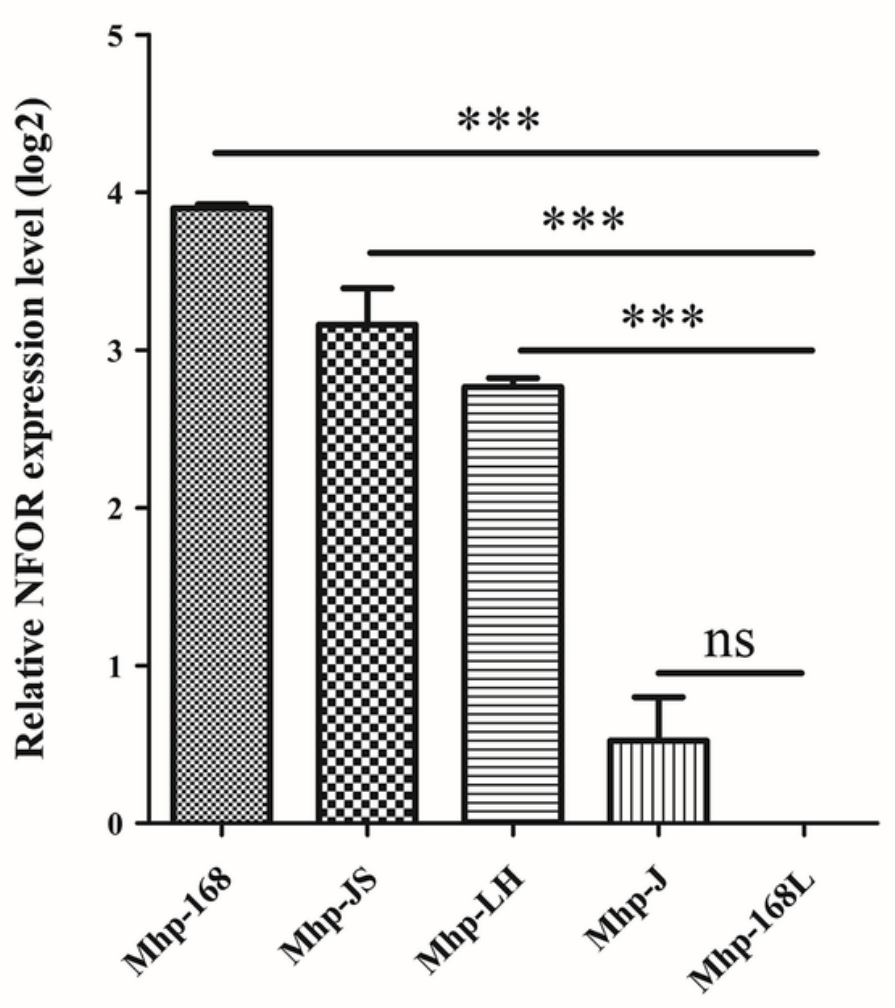

B

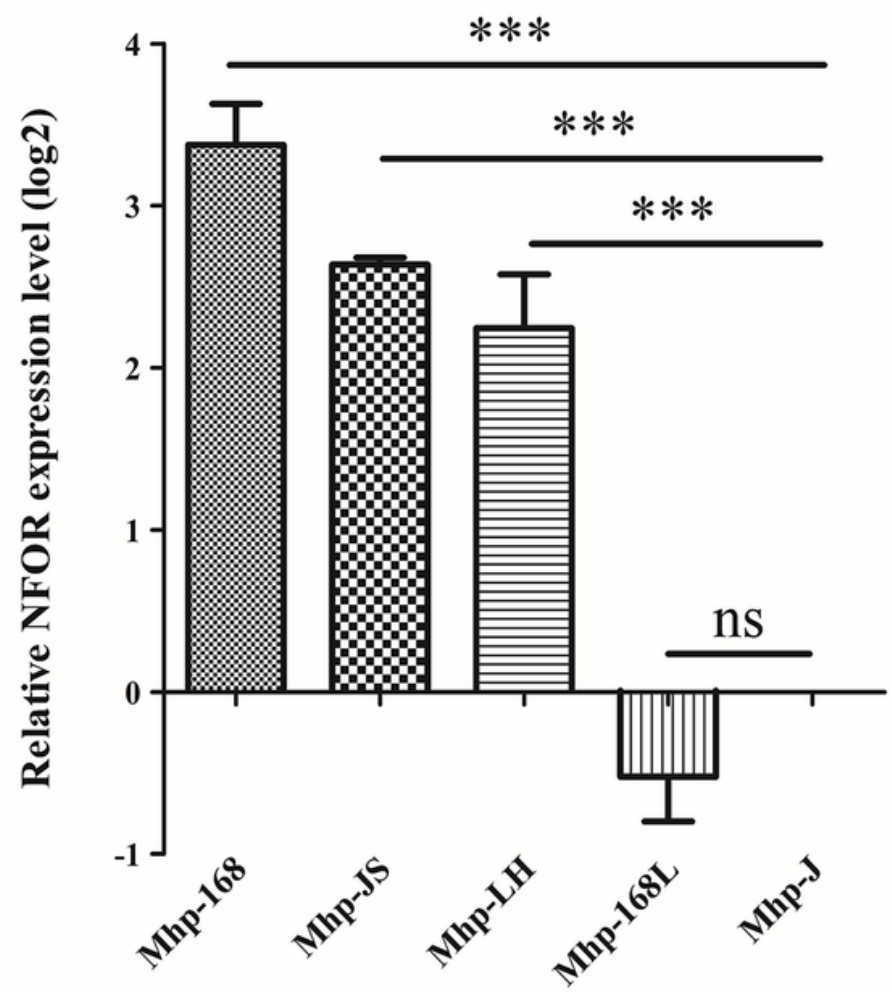

\section{Figure 1}

Relative expression levels of NFOR genes from various Mhp strains differed in virulence compared to the low-virulence Mhp strain 168L (A) and the moderate-virulence Mhp strain J (B) (set to 1). The relative NFOR gene expression level was confirmed by quantitative real-time PCR. Error bars represent standard

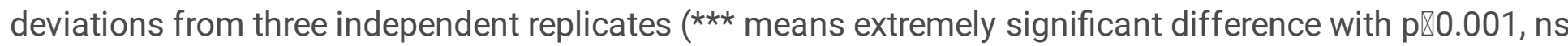
means no significant difference with $\mathrm{p} \otimes 0.05$ ). 


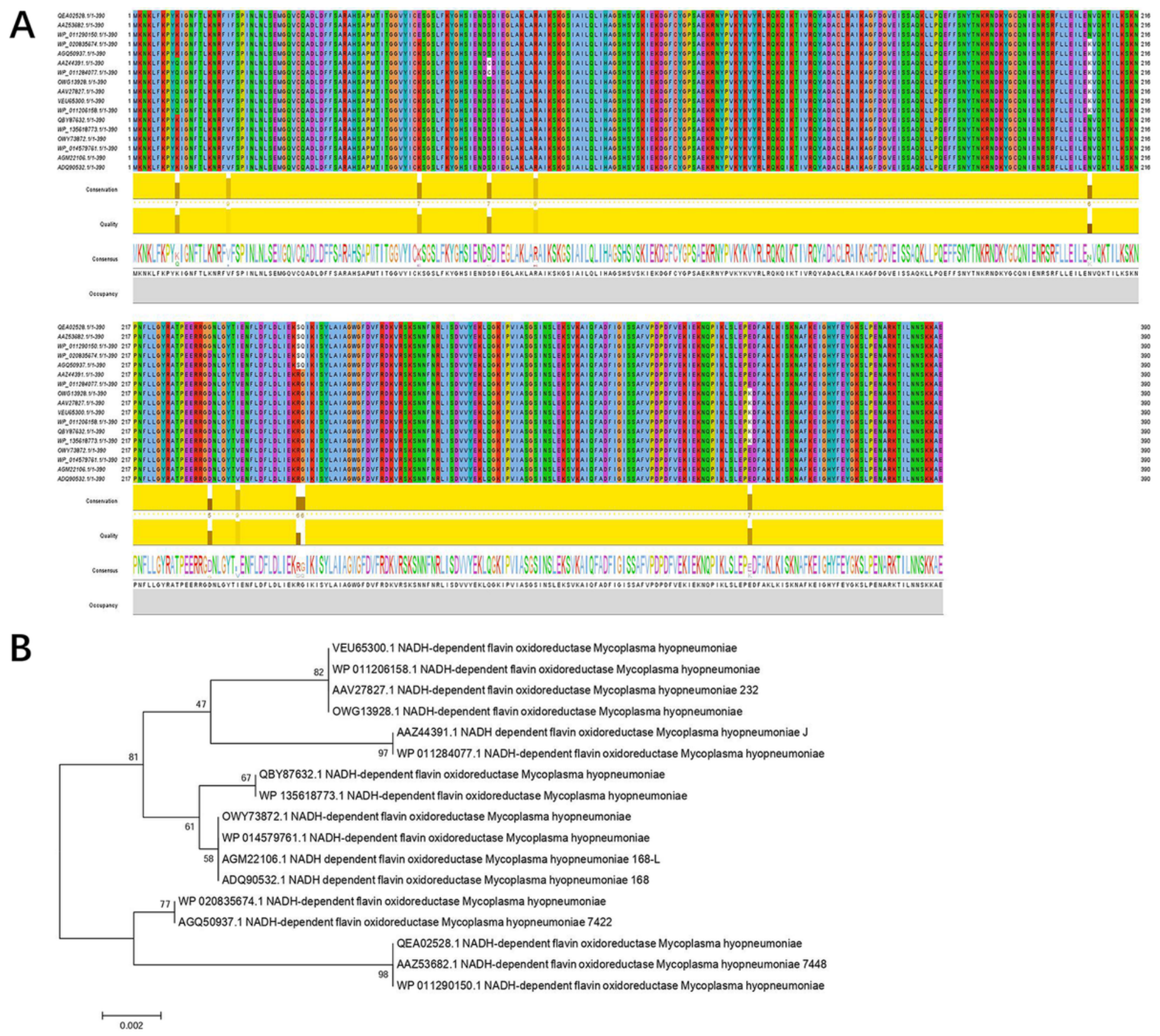

\section{Figure 2}

Multiple sequence alignment and evolutionary analysis of NFOR proteins from various Mhp NFOR protein sequences. (A) Sequence alignment of NFOR proteins from different Mhp strains using Clustal W by JavaView software. Sequences are from the UniProt database, and the consensus is shown with a web logo. (B) Phylogenetic analysis based on the NFOR proteins of different Mhp strains using the neighborjoining $(\mathrm{NJ})$ method with the sequences derived from the NCBI and UniProt databases. The number of phylogenetic branches is expressed as a bootstrap value (2000 replicates) as a percentage of support for each group. The bars represent genetic distance. 

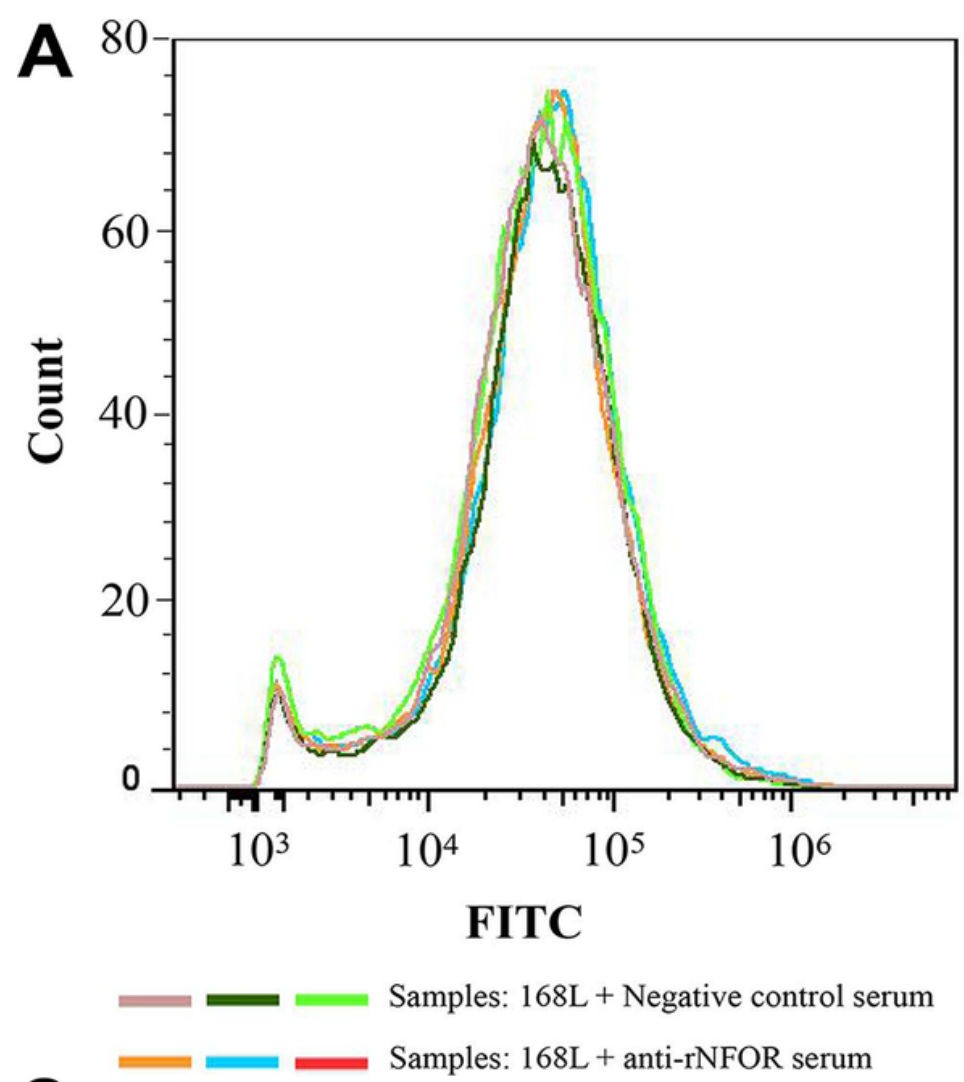
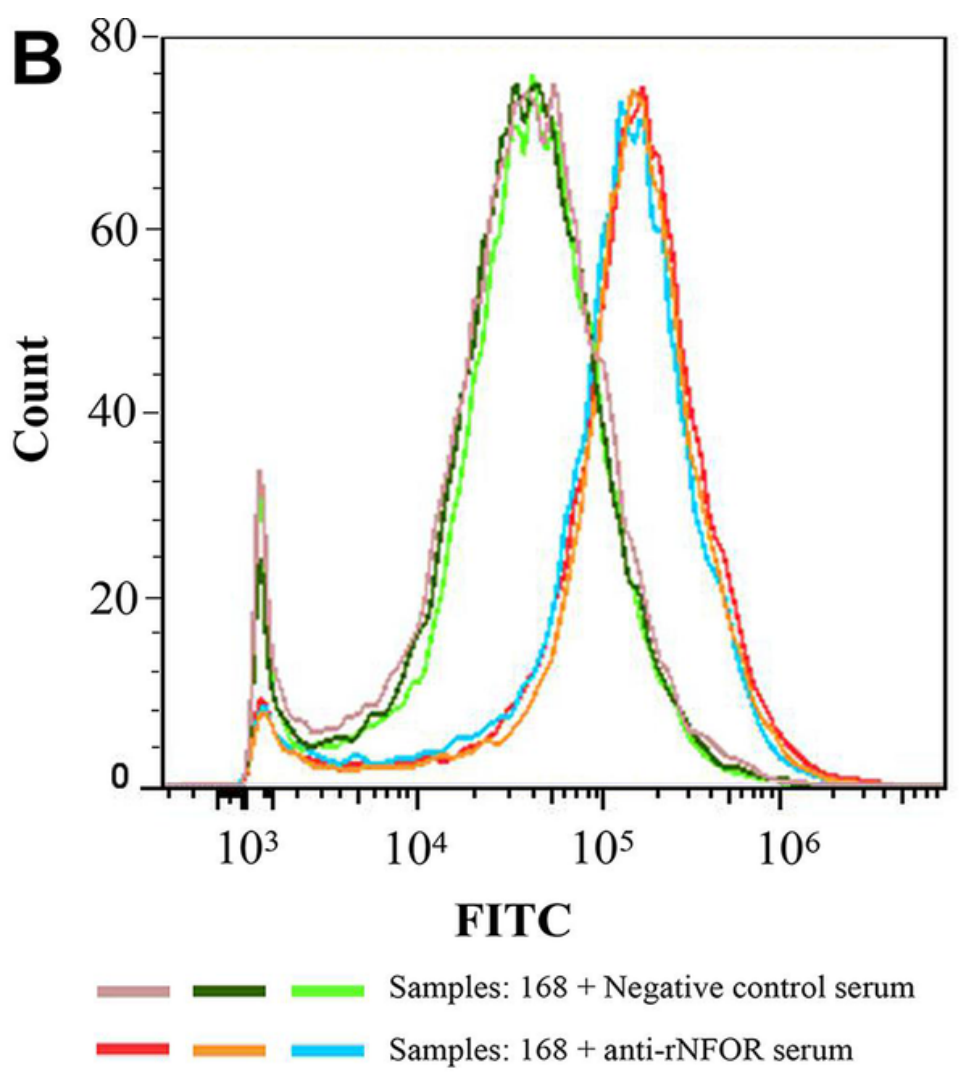
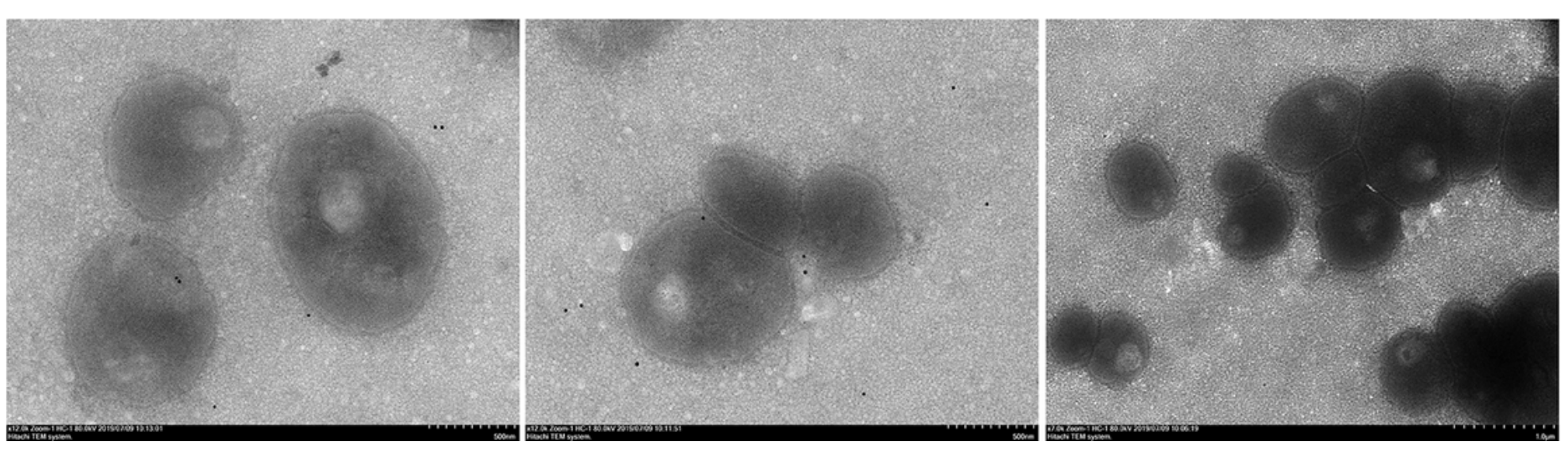

Figure 3

Detection of surface-exposed NFOR by flow cytometry and immunoelectron microscopy techniques. Comparison of the fluorescence intensity of Mhp strains differing in virulence treated with anti-NFOR serum and negative control serum. Fluorescence intensity was assessed by FITC staining with flow cytometry, counting 20,000 cells with three independent replicates. A: Mhp strain $168 \mathrm{~L}$ treated with negative control preimmune serum (negative control) or anti-rNFOR serum; B: Mhp strain 168 treated with negative control preimmune serum or anti-rNFOR serum; C: From left to right are Mhp strains JS and 168 treated with anti-Mhp NFOR and preimmune serum before treatment with secondary gold-conjugated antibodies, respectively. 
A

TRITC

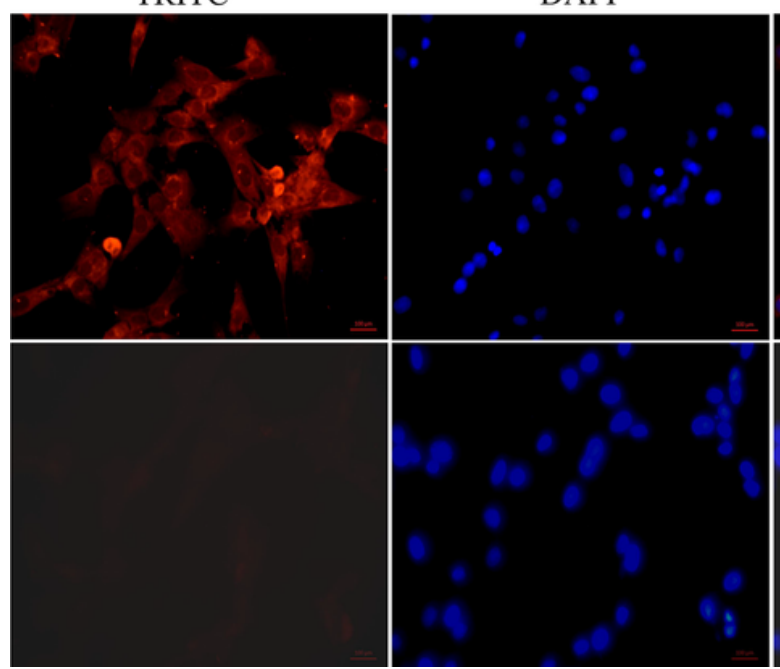

MERGE

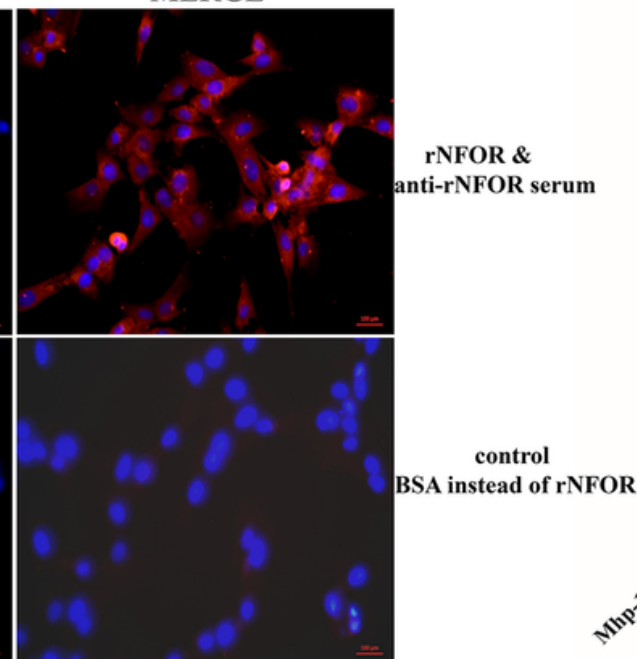

B

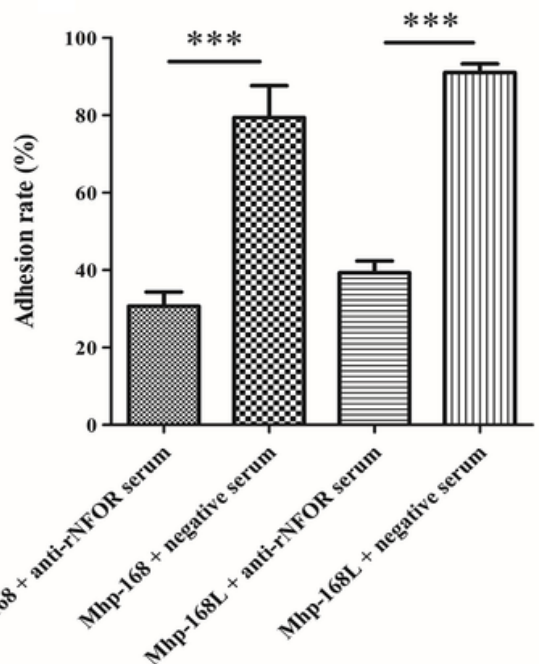

Figure 4

rNFOR adhesion and adhesion inhibition assays using IFA and real-time PCR analysis. (A) hTERT-PBECs were fixed and incubated with purified rNFOR or BSA (control) and anti-rNFOR rabbit polyclonal antibody at a 1:300 dilution (produced in our lab) before incubation with the fluorescent antibody TRITCconjugated anti-IgG (1:100 dilution). TRITC: the anti-rabbit IgG antibody (orange-red) conjugated with TRITC, was used to observe the reactivity of the antibody to IgG; DAPI: 4',6-diamidino-2-phenylindole, the nuclei of all cells were stained with DAPI Reagent (blue); the merging of images is described in the column labeled "merged". The images were captured at 400x magnification. The red bar represents the scale: $100 \mu \mathrm{m}$. (B) Mhp strains 168 and 168L were preincubated with polyclonal antibody against rNFOR or preimmune serum at a dilution of 1:20 before inoculation with hTERT-PBECs. Finally, the adhesion rate was calculated by real-time PCR to count Mhp bacteria. Adhesion rate $=$ (number of Mhp antigens collected from infected cells incubated with anti-rNOX serum/number of Mhp antigens collected from infected cells incubated with preimmune serum) $\times 100$. Quantitative real-time PCR analysis was performed and expressed as log10 DNA copy number per $\mathrm{mL}$ from cell distribution against P97 infected with Mhp. Data are presented as the mean \pm SD from at least three independent replicates ( ${ }^{\star \star \star}$ means extremely significant differences, with $\mathrm{p} \varangle 0.001)$. 


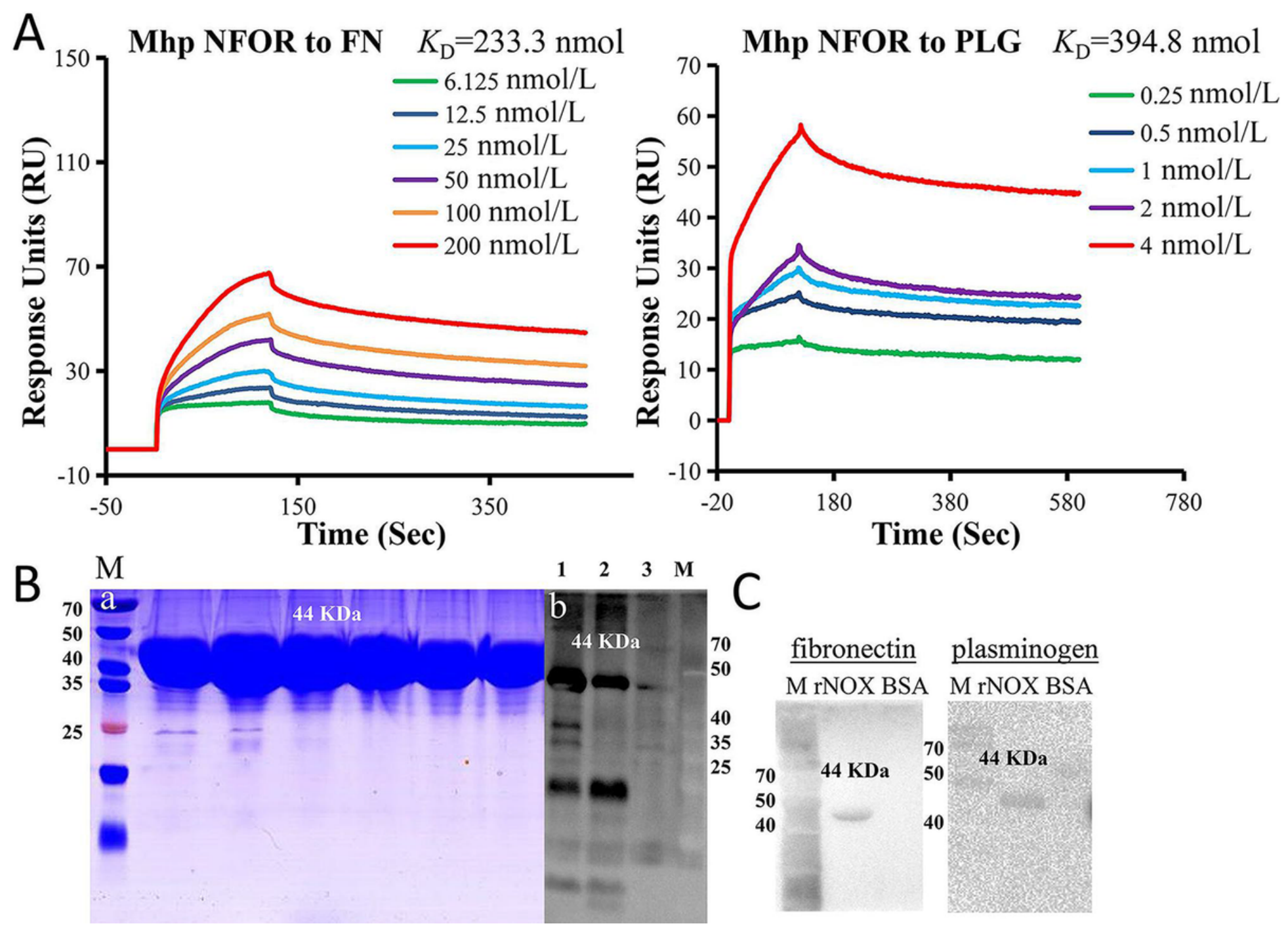

Figure 5

Identification of rNFOR binding ligands. (A) Mhp NFOR in gradient concentrations from $0.25 \mathrm{nmol} / \mathrm{L}$ to 4 $\mathrm{nmol} / \mathrm{L}$ and $6.125 \mathrm{nmol} / \mathrm{L}$ to $200 \mathrm{nmol} / \mathrm{L}$ flow through with immobilized fibronectin and plasminogen in SPR assays. Concentrations of protein are consistent with the colored lines. RU, resonance units. (B) Prokaryotic expression and purification of recombinant protein. WB verification of the prepared polyclonal antibody against Mhp NFOR. "a", Lane M: prestained protein mass markers, with recombinant protein rNFOR having a size of $44 \mathrm{kDa}$. The prepared polyclonal antibody effect of rNFOR was tested by Western blotting. "b", a clear band appeared at the 44-kDa position. Lane 1, unpurified E. coli whole bacterial protein rNFOR. Lanes 2 and 3, purified recombinant protein rNFOR with different loading concentrations (the concentration of Lane 3 was half that of Lane 2). (C) Mhp NFOR with fibronectin and plasminogen interaction analysis by far-Western blot. Lane M: prestained protein mass marker. Lane rNFOR: positive control, Mhp NFOR protein incubated with the anti-rNFOR antibody, fibronectin or plasminogen as well as the anti-fibronectin or anti-plasminogen antibody. Lane BSA: negative control, BSA incubated with the anti-rNFOR antibody, fibronectin or plasminogen as well as the anti-fibronectin or anti-plasminogen antibody. 
A

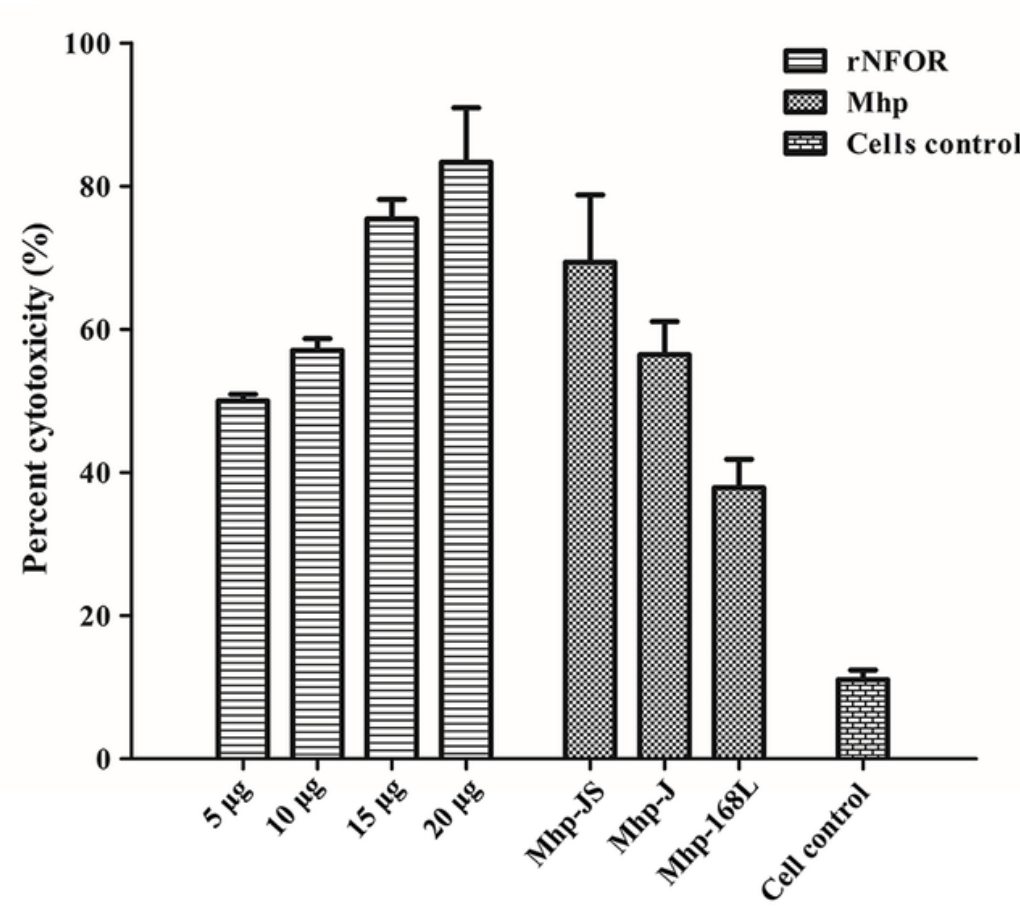

B

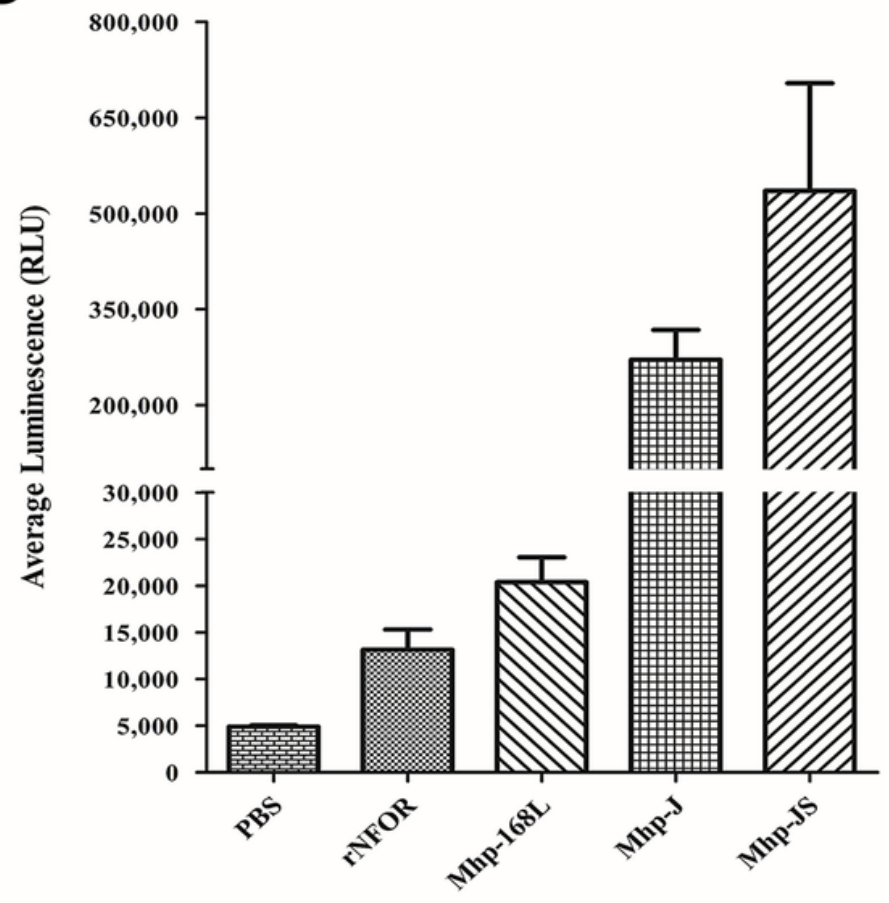

Figure 6

Cytotoxicity and oxidative stress of hTERT-PBECs induced by rNFOR. (A) CytoTox $96 \AA$ Non-Radioactive Cytotoxicity Assay of hTERT-PBECs induced to cytotoxicity after treatment with different concentrations of rNFOR and various Mhp strains differing in virulence (positive control, including the high-virulence strain JS, the moderate-virulence strain $\mathrm{J}$ and the low-virulence strain 168L). (B) ROS-Glo ${ }^{\mathrm{TM}} \mathrm{H} 2 \mathrm{O} 2$ assay of host cell cellular oxidative stress after treatment with rNFOR and different virulent Mhp strains (JS, J and 168L). 

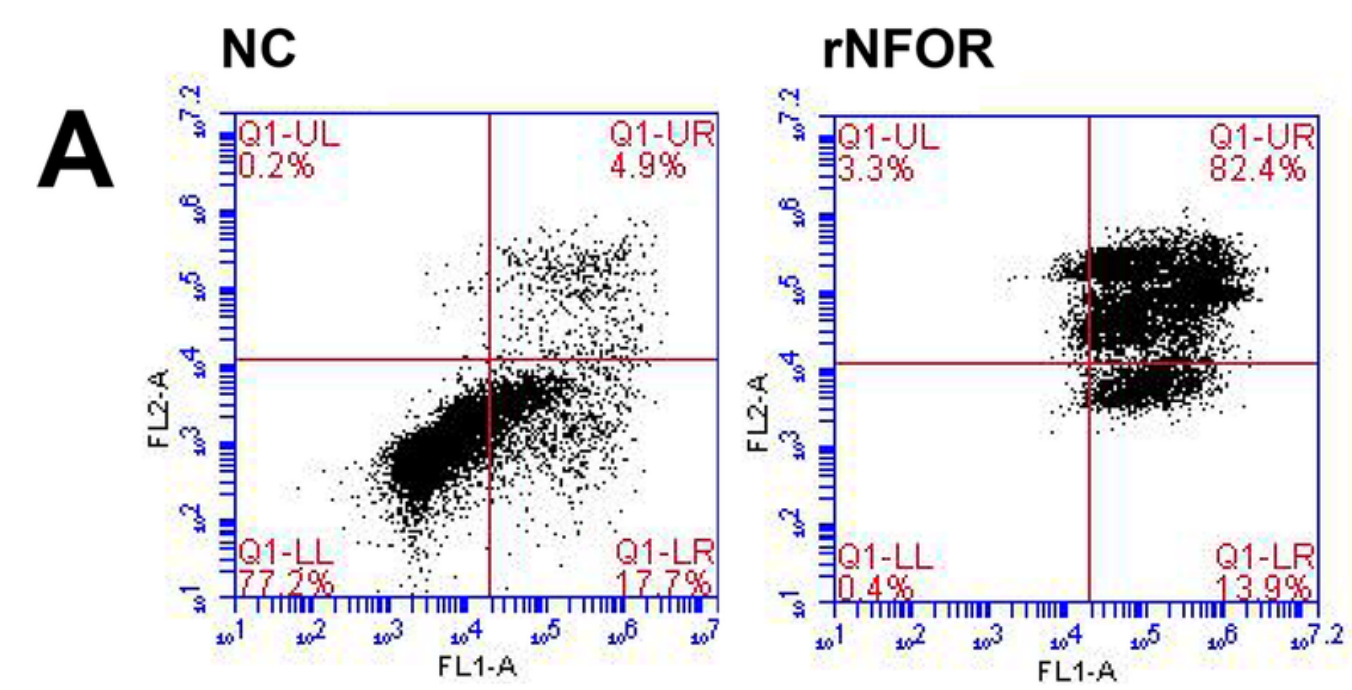

B
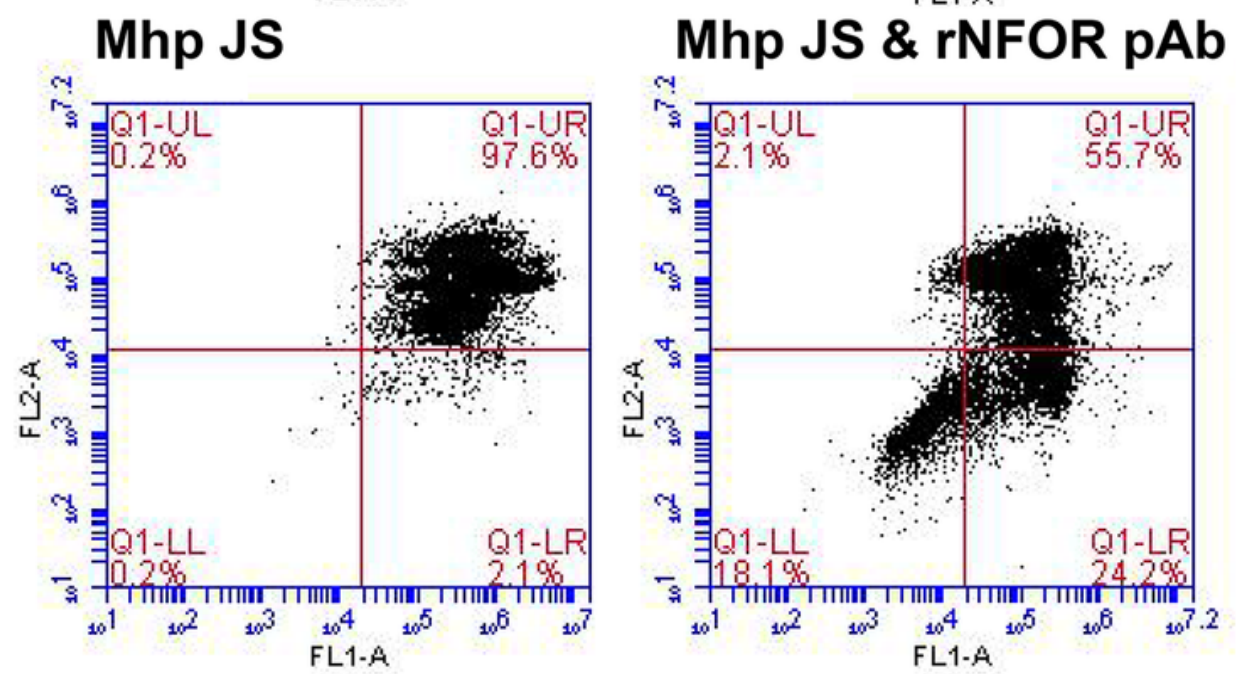

\section{Mhp 168L}

C

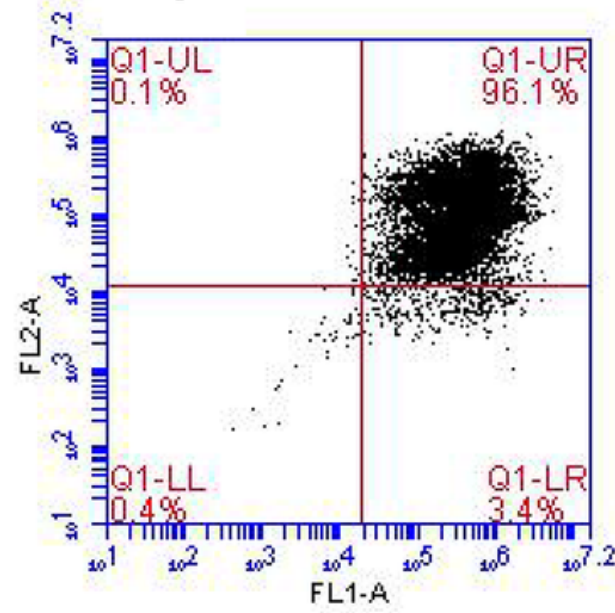

\section{Mhp 168L \& rNFOR pAb}

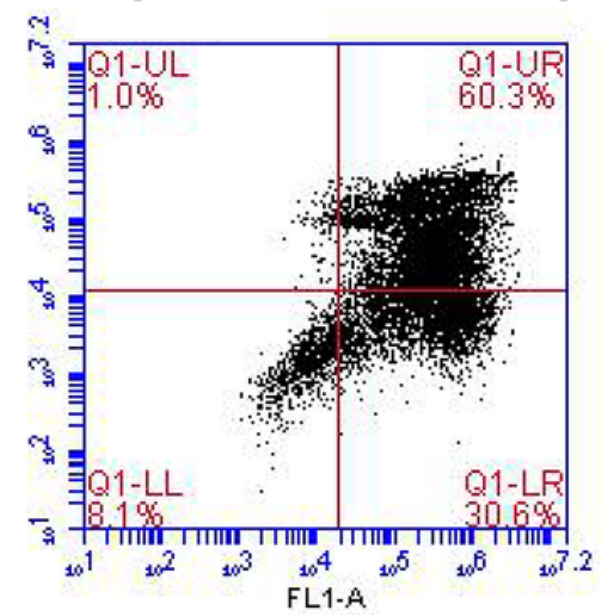

Figure 7

Apoptosis of hTERT-PBECs induced by rNFOR and Mhp strains (high-virulence strain JS and low-virulence strain 168L) was assessed through flow cytometry with the annexin V FITC/PI double staining method 12 $\mathrm{h}$ post infection. The late apoptosis rate (upper right quadrant) showed a large difference. A: Negative cell control, hTERT-PBECs cultured for $12 \mathrm{~h}$ after reaching $80 \%$ confluence in DMEM/F12 without FBA and growth factors before double staining; rNFOR indicates the apoptosis rate of recombinant protein rNOX 
infected with hTERT-PBECs $12 \mathrm{~h}$ post infection before double staining. B: Mhp high-virulence strain JS infected with hTERT-PBECs for $12 \mathrm{~h}$ treated without and with rNFOR polyclonal antibody. C: Mhp lowvirulence strain $168 \mathrm{~L}$ infected with hTERT-PBECs for $12 \mathrm{~h}$ treated without and with rNFOR polyclonal antibody. 